\title{
Role of blue dye for sentinel lymph node detection in early endometrial cancer
}

\author{
Stefano Restaino ${ }^{1 *}$, Carlo Ronsini ${ }^{2}$, Angelo Finelli ${ }^{2}$, Emanuele Perrone ${ }^{3}$, Giovanni Scambia ${ }^{3}$ and Francesco Fanfani ${ }^{2}$
}

\begin{abstract}
Background: Sentinel Lymphonode analysis has become a barely new and innovative way to treat early stages of endometrial cancer (Ballester et al., Lancet Oncol 469-476, 2011; Buda et al., Ann Surg Oncol 2975-81, 2016). Indocyanine green cervical injection is considered gold standard for mapping nodes' drainage. Blue dye is used as a valid alternative in many centers, due to the lower cost of execution. The objective of this video is to prove that methylene blue dye's cervical injection is a valid and "low-cost" method to obtain mapping of lymphatic drainage in patient with early endometrial cancer.

Methods: Fifty-four-year old women, with a recent diagnosis of endometrial cancer IA G2, we performed a radical Hysterectomy type A. We diluted in equal proportions the blue dye and saline and injected $1 \mathrm{cl}$ in depth and $1 \mathrm{cl}$ on the surface of the cervix, at 3 o'clock and 9 o'clock. After 20 min, it was shown with precision the lymphatic drainage until the first lymph node station from both sides.

Results: One external iliac lymph node and one obturator were resected on the left side and one external iliac on the right side. On histological analysis, none of the lymph nodes had any location of metastasis, neither micro-metastasis. Myometrial infiltration was 8/19 mm.

Conclusions: Blue dye cervical injection is a "low-cost", safe, and satisfactory procedure to point out Sentinel Lymph Node of uterus drainage. Other tracers, such as indocyanine green, are widely used in gynecological oncology, but with a higher cost of the product and the needing of a dedicated optical filter to be shown on human view.
\end{abstract}

Keywords: Lymph node, Blue dye, Endometrial cancer

\section{Background}

Sentinel lymph node analysis has become a new and innovative method to treat early stages of endometrial cancer $[1,2]$. In the aim of reducing complications related to lymphoadenectomy, the use of different tracers to identify first lymph node absorber of the drainage chain is widely propagated in gynecological oncology centers. Indocyanine green cervical injection is considered gold standard for mapping nodes' drainage, but this technique requires a dedicated optical filter to catch signal. Blue dye is used as a valid alternative (77 Vs $97 \%$ bilateral detection rate) in many centers, due to the minor cost of performing. The objective of this video is to prove that methylene blue

\footnotetext{
* Correspondence: Restaino.stefano@gmail.com

${ }^{1}$ Gynecological Oncology, Hospital G. Bernabeo, Ortona, CH, Italy

Full list of author information is available at the end of the article
}

dye's cervical injection is a valid and low-cost method to obtain mapping of lymphatic drainage in patient with early endometrial cancer.

\section{Methods}

In a 54-year-old woman, BMI 28, with a recent diagnosis of Endometrial Cancer G2, with no previous imaging suspect of deep myometrial infiltration ( $>50 \%)$, we performed a radical hysterectomy (class A of Querleu-Morrow's classification), with bilateral salpingo-oophorectomy. Furthermore, we decided to act cervical injection of blue dye to identify the first lymph node station. We diluted in equal proportions the blue dye and saline and injected $1 \mathrm{cl}$ in depth and $1 \mathrm{cl}$ on the surface of the cervix, at 3 o'clock and 9 o'clock, in order to obtain a precise mapping of the lymphatic course. As in this case, we prefer to practice injection with a spinal anesthesia needle (27G), for the accurate ratio between length and cross section, which makes 
easier the deep and shallow injection without encountering excessive resistances. Injection of the cervix represents a crucial moment of this method. During this step, indeed, an excess pressure can determine an extravasation of the dye, which would color the entire parametrial space, making it impossible to identify the actual absorber structures. After $20 \mathrm{~min}$, by accessing the retroperitoneum to parametrial level, it was shown with precision the lymphatic course until the first lymph node station from both sides. The lymph nodes had a clear accumulation of tracer, which facilitated the identification even in the absence of appropriate optical filters (Additional file 1).

\section{Results}

One external iliac lymph node and one obturator were resected on the left side and one external iliac on the right side. On histological analysis, none of the lymph nodes had any location of metastasis, neither micro-metastasis. Myometrial infiltration was $8 / 19 \mathrm{~mm}$. Operation time, including cervical injection, was $47 \mathrm{~min}$, and no abnormal bleedings were reported. Patient was discharged the day after.

\section{Conclusion}

In our experience, blue dye cervical injection is a "lowcost", safe, and satisfactory procedure to point out sentinel lymph node of uterus drainage. This could help to avoid an overtreatment such as a systematic pelvic lymphadenectomy in patient which have a diagnosis of uterine malignancy. Other tracers, such as indocyanine green, are widely used in gynecological oncology, but with a higher cost of the product and the needing of a dedicated optical filter to be shown on human view. The recent FIRES study demonstrates that indocyanine green cervical injection has a high degree of diagnostic accuracy in patients with endometrial cancer [3]. Is not in the aim of this case report to prove which is the best tracer for endometrial sentinel lymph node, but it is to prove the feasibility of the method with blue dye, a more "comfortable" product.

\section{Additional file}

Additional file 1: Video. (MP4 32,192 kb)

\section{Funding}

The authors declare that they have no funding

\section{Authors' contributions}

SR contributed to the conception and design. CR helped in drafting the manuscript. AF contributed to the acquisition of data. EM helped in drafting the manuscript. GS carried out the supervision. FF contributed to the conception and design. All authors read and approved the final manuscript.

Consent for publication

We obtained consent to publish.

Competing interests

The authors declare that they have no competing interests.

\section{Publisher's Note}

Springer Nature remains neutral with regard to jurisdictional claims in published maps and institutional affiliations.

\section{Author details}

${ }^{1}$ Gynecological Oncology, Hospital G. Bernabeo, Ortona, CH, Italy.

'Department of Medicine and Aging Sciences University "G. d'Annunzio" of

Chieti-Pescara, Chieti, CH, Italy. ${ }^{3}$ Division of gynecological oncology,

Department of Obstetrics and Gynecology, Catholic University of Sacred

Heart, Rome, RM, Italy.

Received: 15 September 2017 Accepted: 15 November 2017

Published online: 29 November 2017

References

1. Ballester M, Dubernard G, Lecuru F (2011) Detection rate and diagnostic accuracy of sentinel-node biopsy in early stage endometrial cancer: a prospective multicenter study (SENTI-ENDO). Lancet Oncol.:469-476

2. Buda A, Elisei F, Palazzi S (2016) Quality of care for cervical and endometrial cancer patients: the impact of different techniques of sentinel lymph node mapping on patient satisfaction. Ann Surg Oncol:2975-2981

3. Rossi EC, Kowalski LD, Scalici J et al (2017) A comparison of sentinel lymph node biopsy to lymphadenectomy for endometrial cancer staging (FIRES trial): a multicentre, prospective, cohort study. Lancet Oncol:384-392

\section{Submit your manuscript to a SpringerOpen ${ }^{\mathcal{O}}$ journal and benefit from:}

- Convenient online submission

- Rigorous peer review

- Open access: articles freely available online

- High visibility within the field

- Retaining the copyright to your article

Submit your next manuscript at springeropen.com 